\title{
Study of the optical properties of poly(vinyl chloride)-4-[(5-mercapto-1,3,4- thiadiazol-2-yl)diazenyl]phenol complexes
}

\author{
Noora Asaad Witwit \\ Department of Chemistry, College of Science, Al-Nahrain University, Baghdad, 10072, Iraq \\ *Corresponding author at: Department of Chemistry, College of Science, Al-Nahrain University, Baghdad, 10072, Iraq. \\ Tel.: +964.771.1088603. Fax: +964.771.1088603. E-mail address: nona witwit@yahoo.com (N.A. Witwit).
}

\section{ARTICLE INFORMATION}

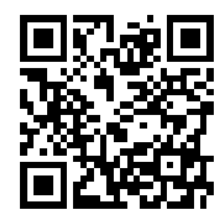

DOI: $10.5155 /$ eurjchem.5.4.652-656.1100

Received: 07 June 2014

Received in revised form: 15 July 2014

Accepted: 03 August 2014

Online: 31 December 2014

\section{KEYWORDS}

PVC

FT-IR

UV-Vis

Thiadizol

Energy gap

Optical properties

\section{Introduction}

Synthesis of polymer-bound chelating ligands and the selective chelation of specific metal ions to bring specific properties to the modified material, such as enhanced thermal stability, multiphase physical responses, compatibility, impact response, flexibility, and rigidity is a field of active research $[1,2]$.

A number of ligands including polydentate amines, crown ethers, phosphines and bipyridines have been bound with mainly poly(styrene-divinyl benzene)copolymers [3,4]. These studies are mostly concerned with ion-binding and catalytic aspects. Very little information appears to exist as to whether, and to what extent, such organic compounds can influence the properties of the macromolecule [5]. Recently, scientists were able to modify PVC, one of the most versatile plastics [6], by introduction aromatic and heterocyclic moieties through halogen displacement reaction [7]. PVC, thus modified, showed improved overall photochemical stability and optical properties [8-10]. The facial chlorine displacement from PVC indicated the possibility on easy anchoring of ligands to PVC matrix and the subsequent synthesis of immobilized transition metal complexes [11]. This article will describe the modification of PVC with anchoring ligand and study its optical properties.

\section{Experimental}

\subsection{Instrumentation}

All the melting points were recorded on a hot stage Gallen Kamp melting point apparatus, and are presented without corrections. The Fourier transform infrared spectroscopy (FTIR) spectra were obtained in the range $4000-400 \mathrm{~cm}^{-1}$ using $\mathrm{KBr}$ disc on FTIR 8300 Shimadzu spectrophotometer. Ultraviolet-visible spectrums were measured using Shimadzu UV-Vis 160 A-Ultraviolet-visible spectrophotometer in range 200-1000 nm. The softening points were determined using thermal microscope (Kofler method), Reichert thermovar. The ${ }^{1} \mathrm{H}$ and ${ }^{13} \mathrm{C}$ NMR spectra were obtained using a Bruker model AC-200P spectrometer $(200 \mathrm{MHz})$. Solvents and starting compounds were Merck or Aldrich and purity confirmed by FTIR.

\subsection{Synthesis of 5-amino-3H-1,3,4-thiadiazole-2-thione (I)}

A mixture of potassium hydroxide $(0.31 \mathrm{~g}, 5.34 \mathrm{mmole})$ and carbon disulfide (5 mL, 5.52 mmole) was dissolved in anhydrous ethanol $(15 \mathrm{~mL})$, later followed by addition of thiosemicarbazide ( $0.5 \mathrm{~g}, 5.49 \mathrm{mmole})$ dissolved in anhydrous ethanol. The reaction mixture was stirred and heated to reflux for $8 \mathrm{~h}$. 


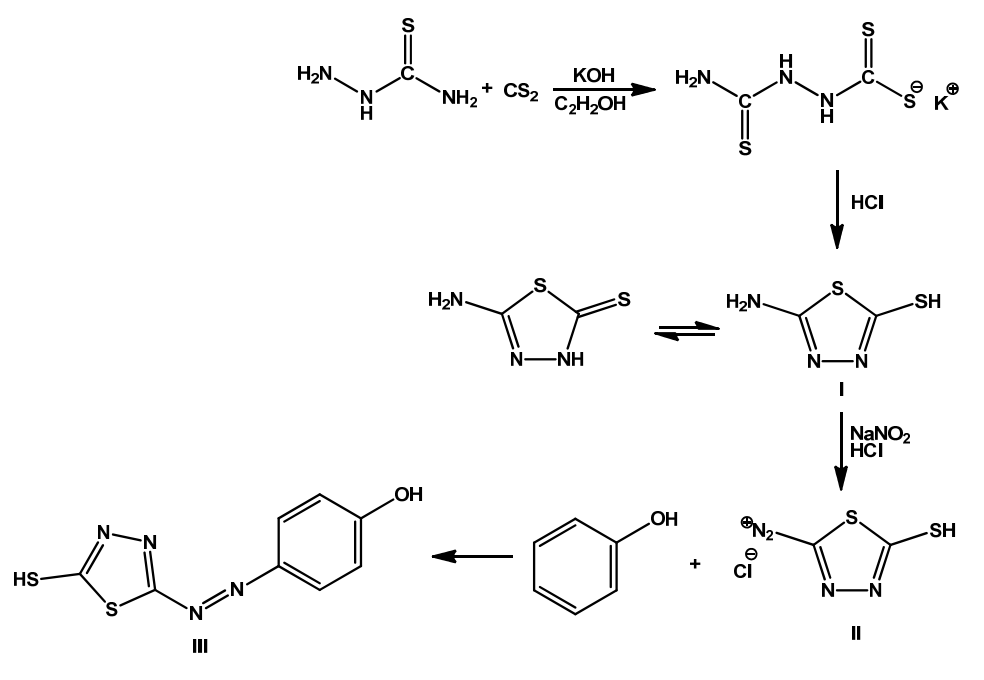

Scheme 1

The ethanol was removed by evaporation in vacuum, and the residue was dissolved in water $(50 \mathrm{~mL})$. This was slowly acidified with $5 \mathrm{~mL}$ of concentrated $\mathrm{HCl}$. The precipitate was filtered to produce compound $\mathbf{I}$. The crude product was washed with cold water, and this yellow solid was recrystallized out of ethanol to give a white solid [12-14] (Scheme 1). Color: White. Yield: $74 \%$. M.p.: $229-231{ }^{\circ} \mathrm{C}$ (Lit.: $232{ }^{\circ} \mathrm{C}$ [15]). ${ }^{1} \mathrm{H}$ NMR $(200$ MHz, DMSO- $\left.d_{6}, \delta, \mathrm{ppm}\right): 7.0\left(\mathrm{~s}, 2 \mathrm{H}, \mathrm{NH}_{2}\right), 13.2(\mathrm{~s}, 1 \mathrm{H}, \mathrm{NH}) .{ }^{13} \mathrm{C}$ NMR (50 MHz, DMSO- $\left.d_{6}, \delta, \mathrm{ppm}\right): 181.2,161.2$. FT-IR (KBr, $v$, $\mathrm{cm}^{-1}$ ): 3340, 3251, 3132, 2925, 1609, 1475, 1362, 1328, 1059. MS (EI, $m / z(\%)): 132.98\left(\mathrm{M}^{+}, 100\right)$. Anal. calcd. for $\mathrm{C}_{2} \mathrm{H}_{3} \mathrm{~N}_{3} \mathrm{~S}_{2}$ : C, 18.03 ;, 2.27 ; N, 31.55. Found: C, 18.00; H, 2.20; N, 31.57\%.

\subsection{Synthesis of 4-[(5-Mercapto-1,3,4-thiadiazol-2-yl) diazenyl]phenol (III)}

To a solution of compound $\mathbf{I}(0.5 \mathrm{~g}, 3.75 \mathrm{mmole})$ in $10 \mathrm{~mL}$ of water, was added $5 \mathrm{~mL}$ of concentrated $\mathrm{HCl}$, which was subsequently diazotized by treatment with sodium nitrate ( $0.31 \mathrm{~g}, 4.49 \mathrm{mmole})$ at $0{ }^{\circ} \mathrm{C}$ with stirring for 20 minutes. The resulting solution of diazonium salt II was slowly added with vigorous stirring at $0-5{ }^{\circ} \mathrm{C}$, over a solution of phenol $(0.42 \mathrm{~g}$, 4.5 mmole) dissolved in sodium hydroxide ( $1 \mathrm{M}, 20 \mathrm{~mL})$. More sodium hydroxide $(20 \mathrm{~mL}, 1 \mathrm{M})$ was added to the reaction mixture and allowed to react for 3 hours. Finally, acidification of the mixture with $1 \mathrm{M} \mathrm{HCl}$ produced an orange colored precipitate, which was filtered off and recrystallized from dimethylformamide:water $(2: 1, v: v)$ to give a dark orange solid as in Scheme 1 [16]. Color: Dark orange. Yield: 45\%. M.p.: 187$189^{\circ} \mathrm{C} .{ }^{1} \mathrm{H}$ NMR $\left(200 \mathrm{Mhz}, \mathrm{CDCl}_{3}, \delta, \mathrm{ppm}\right): 6.97$ (d, $\left.2 \mathrm{H}, \mathrm{Ar}-\mathrm{H}\right)$, $7.83(\mathrm{~d}, 2 \mathrm{H}, \mathrm{Ar}-\mathrm{H})$. FT-IR (KBr, $\left.v, \mathrm{~cm}^{-1}\right): 3100-3500(\mathrm{OH}), 1600$ $(\mathrm{C}=\mathrm{N}), 1535(\mathrm{~N}=\mathrm{N})$. MS (EI, $\mathrm{m} / \mathrm{z}(\%)): 238.00(\mathrm{M}, 100.0 \%)$. Anal. calcd. for $\mathrm{C}_{8} \mathrm{H}_{6} \mathrm{~N}_{4} \mathrm{OS}_{2}$ : C, 40.33; H, 2.52 ;N, 23.52. Found: C, $40.3 ; \mathrm{H}, 2.5 ; \mathrm{N}, 23.5 \%$.

\subsection{Purification of poly(vinyl chloride) [17]}

Commercial poly(vinyl chloride) was freed from additives by re-precipitation from tetrahydrofuran (THF) solution in ethanol. The purified polymer was dried under reduced pressure at room temperature for 24 hours.

\subsection{Synthesis of PVC ligand compound [17]}

A mixture of 0.1 mole of PVC dissolved in THF and 0.05 mole from 4-[(5-mercapto-1,3,4-thiadiazol-2-yl)diazenyl] phenol (III) and 5 drops of pyridine was refluxed for four hours in THF solvent. The precipitated modified polymer separated by evaporated the solvent. Color: Brown. Yield: $69 \%$. Softing p.: 249-253 ${ }^{\circ} \mathrm{C}$. FT-IR (KBr, v, cm-1): 617 (C-Cl), 2912 (C-H), 1535 $(\mathrm{N}=\mathrm{N}), 1360$ (S-C). Anal. calcd. for $\mathrm{C}_{12} \mathrm{H}_{14} \mathrm{~N}_{4} \mathrm{~S}_{2}$ : C, 51.79; H, 5.03; N, 20.14. Found: C, 51.8; H, 5.03; N, 20.14\%.

\subsection{Synthesis of PVC ligand complex [17]}

A general procedure can be adopted for the preparation of all the metal complexes as follows: PVC-L-MII complexes were obtained by dissolving $0.5 \mathrm{~mol}$ of the appropriate transition metal salt in ethanol and dissolving $1.5 \mathrm{~mol}$ of PVC-L dissolved in THF, colored precipitates were obtained then the precipitate was filtered, washed with excess of absolute ethanol. (Scheme 2) shows reactions for the synthesis of PVC-L-MII polymer [18]. The physical data of the prepared PVC-complexes are found in Table 1.

Table 1. Physical data of the prepared complexes.

\begin{tabular}{lll}
\hline Sample & Color & Softing point $\left({ }^{\circ} \mathbf{C}\right)$ \\
\hline PVC-L & Brown & 249 \\
PVC-L-Sn & Yellow & 288 \\
PVC-L-Zn & Reddish brown & 267 \\
PVC-L-Cu & Dark green & 273 \\
\hline
\end{tabular}

\subsection{Films preparation}

A certain concentration of PVC and PVC-L-M $\mathrm{M}^{\mathrm{II}}$ solution (5 $\mathrm{g} / 100 \mathrm{~mL}$ ) in THF was used to prepare polymer films with a thickness of $30 \mu \mathrm{m}$ (measured by a micrometer type $2610 \mathrm{~A}$, Germany). The films were prepared by evaporation technique at room temperature for 24 hours. To remove the possible residual THF solvent, film samples were further dried at room temperature for three hours under reduced pressure. The optical absorbance (A) of the sample was measured as a function of wavelength $(\lambda)$ ranged from 200 to $900 \mathrm{~nm}$ by using computerized Shimadzu UV-VIS 160A-Ultraviolet spectrophotometer full-scale. The light sources are halogen lamp and socket-deuterium lamp. The detector is Si-photodiode and all measurements were performed at room temperature. UV-VIS absorption was made for prepared samples before and after conjunction. The spectra were used to carry out the energy gap by plotting $(\alpha h v)^{2} v s(h v)$ with $r=1 / 2$ which indicates a transition of direct type. The linear portion was best fitted with $r=1 / 2$. Energy gap shift for all samples plotted as a function to conjunction type [19]. 


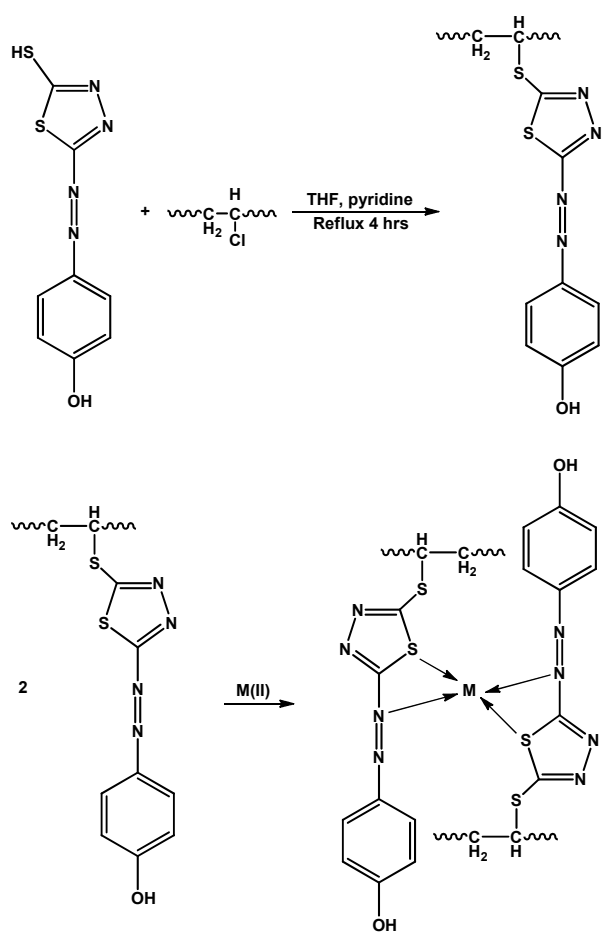

Scheme 2

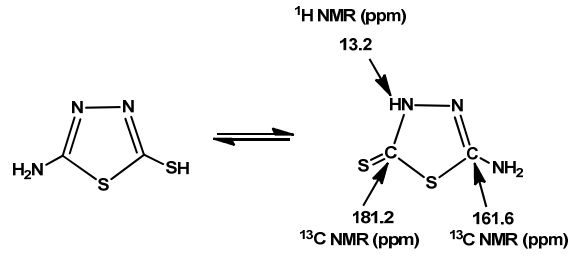

Scheme 3

\section{Result and discussion}

The compound 2-amino-5-thiol-1,3,4-thiodiazol (I) was obtained following a procedure described in the experimental part (Scheme 1). The formation of compound I was confirmed on the basis of a ${ }^{1} \mathrm{H}$ and ${ }^{13} \mathrm{C}$ NMR and FT-IR spectroscopic analysis, showing a stable tautomeric structure for this compound.

The chemical shift of the $\mathrm{C}-2\left({ }^{13} \mathrm{C}\right.$ NMR) in compound I represents the chemical shift characteristic of the carbon atom of a thione group and also in the ${ }^{1} \mathrm{H}$ NMR spectrum a $\mathrm{NH}$ can be observed, representative of a thioamide (13.2-14.0 ppm) instead of a thiol (SH) group (2.0-4.0 ppm). It can therefore be concluded that compound I exists in its thione form in dimethyl sulfoxide solution (Scheme 3).

The most widely practiced reaction of diazonium salts is azo coupling. In this process, the diazonium compound is attacked by coupled to electron-rich substrates. When the coupling partners such phenols, the process is electrophilic aromatic substitution, the aryldiazonium cation is the electrophile and the activated arene is a nucleophile [20].

The modification of PVC with 4-[(5-mercapto-1,3,4-thia diazol-2-yl)diazenyl]phenol (III) was performed by nucleophilic attach of $\mathrm{S}$ atom on the carbon carrying chlorine atom in the polymeric chain followed by departure of chlorine anion as a good leaving group. The structure of the modified polymers was established on the bases of its FT-IR and UV-VIS spectroscopy. Each of the polymers showed band characteristic of its own structure. The modification of PVC with sulphur can be demonstrated by FT-IR spectroscopy, the disappear of the absorption band due to (-SH) stretching frequencies of sulphur clearly indicate the reaction between PVC and sulphur.

UV-VIS technique is used to characterize the prepared polymers in THF as a solvent, the UV-VIS spectra show absorption bands could be attributed to charge transfer and $\pi \rightarrow \pi^{*}$ electronic transitions, these transitions are assigned in the relevance to the structure of the ligand and the modified polymer. As shown in Table 2, the increase of the absorbance in the UV-range for sample of the modified PVC compared with unmodified PVC can be explained by the formation of conjugation double bonds (band corresponding to $\pi \rightarrow \pi^{*}$ transitions) in the modified polymer resulting from introducing aromatic ring thus, the shifting in the absorbance to longer wavelengths (i.e. the bathochromic effect) is a good evidence that modification. The $d$ - $d$ transitions appeared in the visible region. These transitions are assigned in relevance to the structures of complexes [21,22].

The relation between $(\alpha h v)^{2} v s$ photon energy for modified and complex PVC are shown in Figures 1-4 for allowed transition. The shift in the energy gap could be attributed to the formation of polarons and bipolarons. 
Table 2. Absorption and IR spectra of PVC-L and PVC-L-MII.

\begin{tabular}{|c|c|c|}
\hline Complex & Electronic absorption peaks (nm) & Assignment \\
\hline PVC & 261 & $\pi-\pi^{*}$ \\
\hline \multirow[t]{2}{*}{ PVC-L } & 277 & $\pi-\pi^{*}$ \\
\hline & 289 & $\pi-\pi^{*}$ \\
\hline \multirow[t]{4}{*}{$\overline{\text { PVC-L-Cu }}$} & 260 & $\pi-\pi^{*}$ \\
\hline & 266 & $\pi-\pi^{*}$ \\
\hline & 305 & $\pi-\pi^{*}$ \\
\hline & 510 & d-d \\
\hline \multirow[t]{2}{*}{ PVC-L-Sn } & 244 & $\pi-\pi^{*}$ \\
\hline & 267 & $\pi-\pi^{*}$ \\
\hline \multirow[t]{3}{*}{ PVC-L-Zn } & 277 & $\pi-\pi^{*}$ \\
\hline & 285 & $\pi-\pi^{*}$ \\
\hline & 320 & $\pi-\pi^{*}$ \\
\hline Complex & IR $\left(\mathrm{cm}^{-1}\right)$ & Assignment \\
\hline \multirow[t]{2}{*}{$\overline{\mathrm{PVC}}$} & 614 & C-Cl stretching \\
\hline & 2910 & C-H stretching \\
\hline \multirow[t]{4}{*}{$\overline{\mathrm{PVC}-\mathrm{L}}$} & 617 & $\mathrm{C}-\mathrm{Cl}$ \\
\hline & 2912 & $\mathrm{C}-\mathrm{H}$ \\
\hline & 1535 & $\mathrm{~N}=\mathrm{N}$ \\
\hline & 1360 & S-C \\
\hline \multirow{6}{*}{ PVC-L-Cu } & 2910 & C-H (alphatic) \\
\hline & 623 & $\mathrm{C}-\mathrm{Cl}$ \\
\hline & 1520 & $\mathrm{~N}=\mathrm{N}$ \\
\hline & 1330 & S-C \\
\hline & 420 & $\mathrm{Cu}-\mathrm{S}$ \\
\hline & 524 & $\mathrm{Cu}-\mathrm{N}$ \\
\hline \multirow{6}{*}{ PVC-L-Sn } & 2912 & C-H (alphatic) \\
\hline & 615 & $\mathrm{C}-\mathrm{Cl}$ \\
\hline & 1512 & $\mathrm{~N}=\mathrm{N}$ \\
\hline & 1328 & C-S \\
\hline & 442 & Sn-S \\
\hline & 559 & Sn-N \\
\hline \multirow[t]{7}{*}{$\overline{\text { PVC-L-Zn }}$} & 2914 & C-H (alphatic) \\
\hline & 615 & $\mathrm{C}-\mathrm{Cl}$ \\
\hline & 1525 & $\mathrm{~N}=\mathrm{N}$ \\
\hline & 522 & $\mathrm{Zn}-\mathrm{N}$ \\
\hline & 1300 & C-S \\
\hline & 436 & Zn-S \\
\hline & 522 & $\mathrm{Zn}-\mathrm{N}$ \\
\hline
\end{tabular}

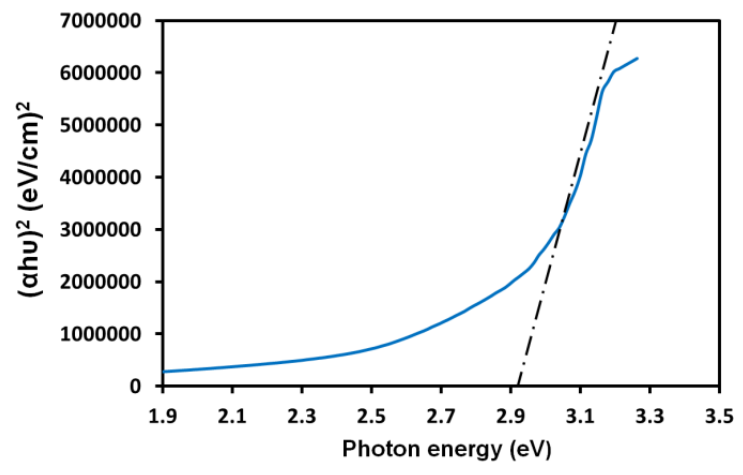

Figure 1. Allowed direct transition $(\alpha h v)^{2} v s$ energy for PVC-L.

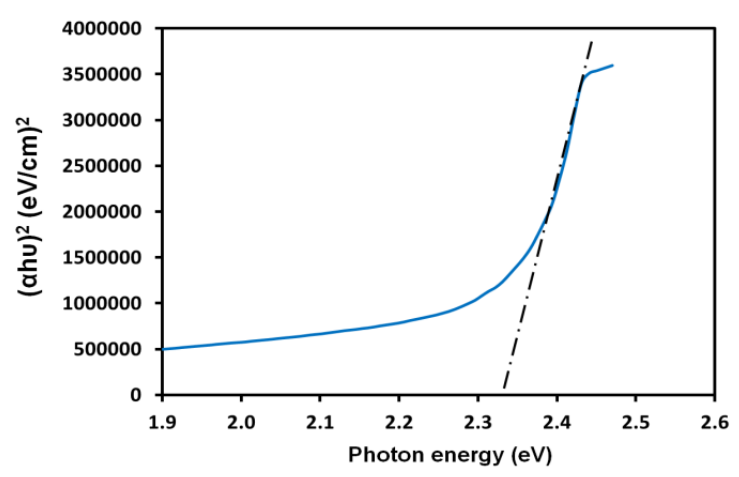

Figure 2. Allowed direct transition $(\alpha h v)^{2} v s$ energy for PVC-L-Sn ${ }^{\mathrm{II}}$.

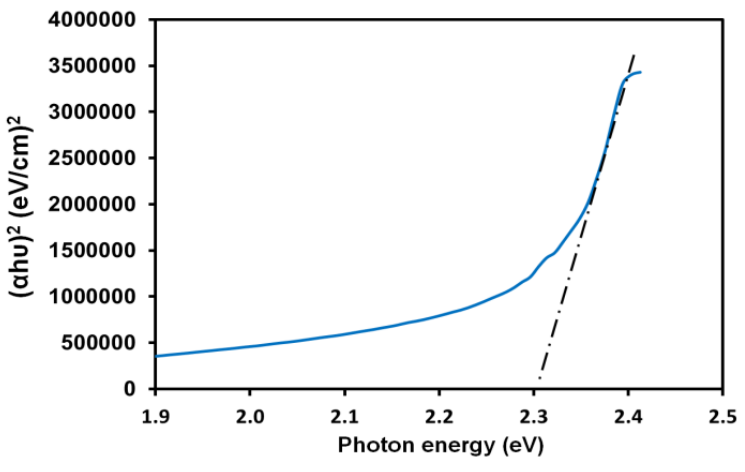

Figure 3. Allowed direct transition $(\alpha h v)^{2} v s$ energy for PVC-L-Zn"I.

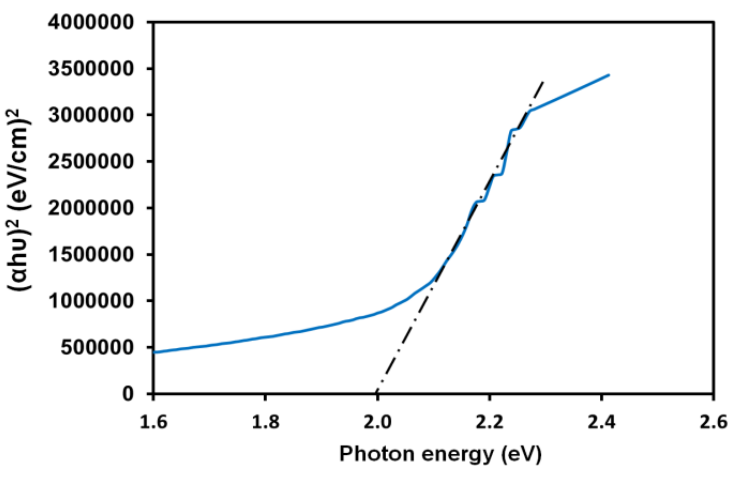

Figure 4. Allowed direct transition $(\alpha h v)^{2} v s$ energy for PVC-L-CuII. 
The evidence of polar on formation is made that the reaction in band to band transitions due to the shifting the band density of state toward the energy gap. This observation is not like doping of conventional semiconductor when the band to band absorption strength dose not affected by the formation of dopant state in the energy gap. The effect of modification and complex formation on the values of activation energies 1 was investigated and the results shown in Table 3. The results presented in Figures 1-4 indicate the existence of two bipolaron bands in the energy gap. The first one represents the transition from valance band to bonding bipolaron band. The second band represents the transition from valance band to anti- bonding bipolaron band which is in a good agreement with results are obtained by other workers [23]. Conductivity measurement of PVC with additive can obtain by adopting the data of energy gap [24,25], (see Table 3 and Figures 1-4). The conductivity measurement for PVC in the presence of additive increase in the following order: PVC-L-Cu > PVC-L-Zn > PVC-LSn $>$ PVC-L > PVC [17].

Table 3. Indicate the energy band gap according to the direct allowed transition.

\begin{tabular}{ll}
\hline Sample & $\boldsymbol{E}_{\mathrm{g}}(\mathbf{e v})$ \\
\hline PVC & 5.79 \\
PVC-L & 2.91 \\
PVC-L-Sn & 2.33 \\
PVC-L-Zn & 2.30 \\
PVC-L-Cu & 2.00 \\
\hline
\end{tabular}

\section{Conclusion}

Azo compounds were synthesized, which were derived from heterocyclics and basic phenolic-type structures. Possibilities for synthesis with more complex structures were certainly possible, and their applications are yet to be developed and were synthesized from reaction of diazonium salts is azo coupling with phenol. PVC bound 4-[(5-mercapto1,3,4-thiadiazol-2-yl)diazenyl]phenol can be synthesized by Chlorine displacement reaction between PVC and L in alkaline condition. Subsequent complexation of PVC-L with $\mathrm{Cu}^{\mathrm{II}}, \mathrm{Zn}^{\mathrm{II}}$, and $\mathrm{Sn}^{\mathrm{II}}$ yield intensely colored PVC-L-MII complexes those exhibit improved overall optical properties. The energy gaps were measured as behavior of conductivity and it is conceded that the energy for PVC is affected by modification and complexation.

\section{Acknowledgement}

Gratefully acknowledged the funding support from The Chemistry Department in College of Science, Al-Nahrain University, Iraq.

\section{References}

[1]. Amit, B.; Rawlins, J. W.; Ray, P. Polymer Crafting and Crosslinking. John Wiley \& Sons Inc., 2009.

[2]. Chen, Y.; Song, Y.; Zhang, Y.; Lang. Inorg. Chem. Commun. 2008, 11, 572-575.

[3]. Gustafsson, B.; Hakansson, M.; Jagner, S. Inorg. Chim. Acta 2003, 350, 209-214.

[4]. Genhua, Z.; Pan, C. Polymer 2005, 46, 2802-2810.

[5]. Jumat, S.; Salah, N.; Ahmed, A.; Yousif, E. Chem. Tech. Res. 2011, 3(2), 981-987.

[6]. Moulay, S. Progr. Polymer Sci. 2010, 35, 303-331.

[7]. Claudiu, L.; Edina, R.; Bogdan, M.; Teodora, Z.; Gheorghe, H. U. P. B. Sci. Bull. Series B 2010, 72, 127-140.

[8]. Yousif, E.; Hameed, A.; Bakir, E. J. Al-Nahrain Univ. (Science) 2007, 10, 7-12.

[9]. Mohammed, A.; Yousif, E.; Alwash, A.; Yang, F. Iraqi J. Polymers 2009, 13(2), 27-36.

[10]. Yousif, E.; Hameed, A.; Kamil, A.; Farina, Y.; Asaad, N.; Graisa, A. Aust. J. Basic Appl. Sci. 2009, 3(3), 1786-1794.

[11]. Aliwi, M.; Yousif, E; Otaiwi, A. Iraqi J. Polymers 2005, 10(1), 53-64.

[12]. Guha, P. C. J. Am. Chem. Soc. 1922, 44, 1510-1517.

[13]. Kumar, S.; Kaushik, D.; Bawa, S.; Khan, S. Chem. Biol. Drug Design 2012, 79(1), 104-111.
[14]. Gaber, M.; El-Sayed, Y. S.; El-Baradie, K. Y.; Fahmy; R. M. Spectrochim. Acta A 2013, 107, 395-370.

[15]. Abdel-Rahman T. M. Phosphorus Sulfur 2006, 181(8), 1737-1754

[16]. Pedro, O. L.; Leonel, V.; Claudio, A.; Miguel, G.; Pedro, O. L. J. Chil. Chem. Soc. 2007, 52(1), 1120-1122.

[17]. Abdallh, M. J. Al-Nahrain Univ. (Science) 2013, 16(2), 24-29.

[18]. Ahmed, A.; Najim, T.; Salimon, J.; Salih, N.; Graisa, A.; Farina, Y.; Yousif, E. ARPN-J. Eng. Appl. Sci. 2010, 5, 43-45.

[19]. Yousif, E.; Adel, H.; Moued, F. J. Appl. Sci. Res. 2010, 6(7), 879-882.

[20]. Smith, Michael B.; Jerry, M. Advanced Organic Chemistry, $6^{\text {th }}$ edition, New York: Wiley, 2007.

[21]. Al-Ramadan, Y. Optical Mater. 2000, 14, 287-290.

[22]. Yousif, E. J. King Saud Univ. 2012, 24, 167-170.

[23]. Al-Daeif, Y.; Yousif, E.; Abdul Nabi, M. J. Al-Nahrain Univ. (Science) 2012, 15(2), 79-83.

[24]. Salimon, J.; Salih, N.; Ahmed, A.; Yousif, E. Int. J. ChemTech Res. 2011 3(2), 981-987.

[25]. Yousif, E.; Abdallh, M.; Hashim, H.; Salih, N.; Salimon, J.; Abdallh, B. M.; Win, Y. Int. J. Ind. Chem. 2013, 4(4), 1-8. 